Instituto Internacional de Investigación y Desarrollo Tecnológico Educativo INDTEC, C.A. DOI: $\mathrm{https://doi.org/10.29394/Scientific.issn.2542-2987.2018.3.10.1.20-39}$

OAI-PMH: http://www.indteca.com/ojs/index.php/Revista Scientific/oai

Artículo Original / Original Article

\title{
Necesidades de Capacitación de los Docentes de Educación Inicial sobre las Altas Capacidades Intelectuales
}

\author{
Autora: Adriana Josefina Ovalles Villegas \\ Universidad Pedagógica Experimental Libertador, UPEL \\ aovalles40@gmail.com \\ Barinas, Venezuela
}

\section{Resumen}

Los sujetos con Necesidades Educativas Especiales son aquellos niños, jóvenes y adultos que por sus características intelectuales, sensoriales y físicas presentan interferencias en el aprendizaje; en ese sentido, en Venezuela, la modalidad de educación especial para garantizar cierta homogeneidad ha creado siete áreas de atención: Deficiencias Visuales, Deficiencias Auditivas, Retardo Mental, Autismo, Impedimentos Físicos, Dificultades de Aprendizaje y Talento Superior. En cuanto a esta última, los niños, niñas y jóvenes con talento superior 0 altas capacidades intelectuales requieren planes y programas especializados para brindarle la atención educativa según sus condiciones. El contexto problemático es el vacío existente en la atención educativa de este tipo de infantes, por lo que resulta importante indagar acerca del conocimiento que poseen los docentes del nivel de educación inicial sobre esta condición. El factor relevante es que los niños con altas capacidades intelectuales o con talento no presentan características o necesidades homogéneas, sino que, en cambio, son tan distintas entre sí como el resto de sus compañeros; sus requerimientos educativos son distintos. De allí que el propósito de este artículo es determinar las necesidades de capacitación del docente de educación inicial sobre las altas capacidades intelectuales. En cuanto a la modalidad de investigación es descriptiva, con un diseño de campo no experimental, utilizándose un cuestionario en escalas alternativas impares, validado a través del juicio de experto y la confiabilidad se realizó con el coeficiente Alfa de Cronbach. Entre los hallazgos se tiene que el $55,56 \%$ manifiestan que no manejan la información sobre el tema, así como el $61,11 \%$ revelan que desconocer el método para diagnosticar y en cuanto a las estrategias didácticas para la atención educativa el $83,33 \%$ de las encuestadas relatan que no las conocen. Se recomienda establecer condiciones educativas favorables a la estimulación, creando elementos de intervención y no solo de identificación.

Palabras clave: competencias del docente; talento; educación especial; educación de la primera infancia. 


\title{
Training Needs of Initial Education Teachers on High Intellectual Capacities
}

\begin{abstract}
The subjects with Special Educational Needs are those children, youth and adults that due to their intellectual, sensory and physical characteristics present interferences in the learning; In that sense, in Venezuela, the special education modality to guarantee a certain homogeneity has created seven areas of attention: Visual Impairments, Auditory Deficiencies, Mental Retardation, Autism, Physical Impairments, Learning Difficulties and Superior Talent. Regarding the latter, children and young people with superior talent or high intellectual abilities require specialized plans and programs to provide educational attention according to their conditions. The problematic context is the existing vacuum in the educational attention of this type of infants, so it is important to inquire about the knowledge that teachers have of the initial education level about this condition. The relevant factor is that children with high intellectual capacities or with talent do not present homogeneous characteristics or needs, but instead, they are as different from each other as the rest of their classmates; their educational requirements are different. Hence, the purpose of this article is to determine the training needs of the initial education teacher on the high intellectual capacities. Regarding the research modality, it is descriptive, with a non-experimental field design, using a questionnaire in odd alternative scales, validated through expert judgment and reliability with the Cronbach's Alpha coefficient. Among the findings, 55.56\% state that they do not handle the information on the subject, as well as $61.11 \%$ reveal that they do not know the method to diagnose and in terms of teaching strategies for educational attention $83.33 \%$ of respondents report that they do not know them. It is recommended to establish educational conditions favorable to stimulation, creating elements of intervention and not just identification.
\end{abstract}

Keywords: teacher qualifications; talent; special education; early childhood education.

Date Received: 04-05-2018

Date Acceptance: 25-06-2018 


\section{Introducción}

El Ministerio de Educación (1997): señala que los sujetos con Necesidades Educativas Especiales (NEE), "son aquellos cuyas características de naturaleza variable, físicas, psicológica o social, requieren de medidas particulares en su favor, ya sea durante toda su vida o en un momento de la misma" (pág. 7); clasificándose estas necesidades según los tipos de deficiencias o dificultades, permitiendo así garantizar cierta homogeneidad, estas son: Deficiencias Visuales, Deficiencias Auditivas, Retardo Mental, Autismo, Impedimentos Físicos, Dificultades de Aprendizaje y Talento Superior. Esta última área, Talento Superior o Altas Capacidades Intelectuales, es sobre la cual se dirige el presente artículo.

Con relación a lo anterior, en algunos niños con Altas Capacidades Intelectuales, se genera ciertos problemas en el entorno social, por lo que se requiere brindarle una atención especializada según su potencial individual, proveyéndoles la atención educativa que necesita según sus características individuales. Al respecto:

es preciso no olvidar que si los niños superdotados o con talento no reciben de forma oportuna una atención educativa a sus capacidades y necesidades específicas, también pueden presentar dificultades de aprendizaje o alteraciones en la personalidad y del comportamiento (Blanco, Ríos y Benavides, 2004, pág. 49).

En tal sentido, se pretendió determinar la necesidad de capacitación que tienen las docentes del Centro de Educación Inicial Los Retoñitos, ubicado en la Parroquia Alto Barinas del Municipio Barinas del estado Barinas sobre las Altas Capacidades Intelectuales en los preescolares de 5 y 6 años de edad.

\section{El Problema}

\subsection{Planteamiento del Problema}

El presente artículo está orientado hacia la detección de la necesidad 
de capacitación que presentan las docentes de educación inicial en el área de talento o Altas Capacidades Intelectuales ( $\mathrm{ACl}$ ), el cual engloba la variabilidad individual de este colectivo, así como la superdotación intelectual, talento y precocidad intelectual. De igual manera, el uso de la terminología apropiada se apoya en los estudios de Mönks y Mason (2000), citados por Benavides, Maz, Castro y Blanco (2004a), quienes "tratan los siguientes términos como sinónimos: dotado (gifted), altamente capaz (highly able) y talentoso (talented)" (pág. 144).

En tal sentido, cuando se habla de sujeto con Altas Capacidades Intelectuales $(\mathrm{ACl})$, se refiere a aquellos individuos que poseen un desarrollo cognitivo superior al de su grupo erario, y requieren de atención especializada que permitan brindarle la atención educativa según sus condiciones, tal como plantea la UNESCO (1994), citado por Benavides, Maz, Castro y Blanco (2004b):

Cada niño tiene características, intereses, capacidades y necesidades que le son propias; si el derecho a la educación significa algo, se deben diseñar los sistemas educativos y desarrollar los programas de modo que tengan en cuenta toda la gama de esas diferentes características y necesidades (pág. 9).

Con relación a lo anterior, no solo por el hecho de poseer un potencial se garantiza el éxito, es imprescindible guiar el camino a seguir, su proceso de crecimiento. Adicionalmente, como lo manifiestan Matos, Vethencourt, Peña y Briceño (2018): "la inteligencia no es permanente, es alterable; en otras palabras, es que cada persona no posee el mismo nivel de inteligencia con el que nace y, la inteligencia no es unitaria, puede manifestarse en diferentes formas" (pág. 253), y este potencial superior puede desarrollarse o eliminarse.

Es conveniente destacar en este punto, que en algunas ocasiones, un niño o niña que no tiene las mejores calificaciones, puede poseer Altas Capacidades Intelectuales (ACl). Así mismo, pueden ser erróneamente 
diagnosticados como hiperactivos o incluso con déficit de atención, aunque Barrios, (2009a), asegura que:

una niña o u niño hiperactivo, incluso con déficit de atención puede ostentar un cociente intelectual superior a 130. Los niños que realmente presentan "Déficit de atención e hiperactividad" se caracterizan porque sólo pueden estar concentrado en algo que les guste o les interese por un promedio de 3 a 5 minutos, mientras que los superdotados ante la misma situación pueden durar quietos más de 30 min., llegando a durar horas inmersos en actividades que les gusta (pág. 5).

Por todo lo expresado anteriormente, las docentes de educación inicial requieren de herramientas que les permita identificar a los preescolares quienes pudiesen presentar un potencial cognitivo superior y así aplicar estrategias de trabajo diseñadas especialmente para las diferentes potencialidades e intereses existentes en el aula; como lo plantea Barrios (2009b):

...con adecuadas bases científicas, académicas y psicológicas se capaciten para facilitar el pleno desarrollo de las potencialidades de estos niños, sobre todo se les puede motivar al desarrollo de sus potenciales talentos mediante la estimulación de las inteligencias múltiples y mediante un programa de aprendizaje autodirigido con el uso de herramientas de telemáticas (pág. 6).

Otro punto referencial es el señalado por Freeman (1988): quien plantea que "hay dos características que comparten y que les diferencian del resto de los alumnos: Aprenden más rápidamente, y tienen mayor profundidad y extensión en el aprendizaje" (pág. 54).Estas características deben tenerse presentes en el momento de realizar la identificación temprana con el propósito de disminuir en lo más posible cualquier déficit intelectual, procurando el máximo desarrollo potencial de los sujetos, en este sentido, Benito (2008a): considera que "la edad óptima para la identificación de un niño como superdotado es entre los 4 y los 7 años" (pág. 4), no con el propósito de 
etiquetarlos sino contribuir con el desarrollo de su potencial.

En este sentido, Artiles (2006), sugiere que:

.... es preciso iniciar y regular la detección de forma precoz para evitar que los alumnos de los primeros niveles con altas capacidades intelectuales no sean convenientemente estimulados en la familia (sobre todo en las familias desfavorecidas) y en la escuela, limitando el desarrollo de su potencial intelectual y, por lo tanto, su derecho al crecimiento pleno como persona. Si no se estimulan los microprocesos intelectuales de este alumnado, ya presentes en edades tempranas, se perderían e incluso podrían degenerar en inadaptaciones escolares y fracasos en el rendimiento (pág. 36).

En consecuencia, es importante realizar una indagación de los niños con altas capacidades intelectuales, en la edad señalada debido a que es cuando más se desarrolla su inteligencia y sus capacidades, adicionalmente, en estas edades disfrutan mostrar de lo que son capaces de hacer y de aprender.

Desde esa perspectiva, en las aulas del Centro de Educación Inicial Los Retoñitos, ubicado en la Parroquia Alto Barinas del Municipio Barinas del estado Barinas, se infiere que los procesos de enseñanza y aprendizaje van dirigidos hacia niños con capacidades dentro de la media; de hecho, en entrevistas informales realizadas con el personal docente y directivo de la referida institución educativa, ellos manifiestan verbalmente que las actividades propias están dirigidos a estudiantes promedios. De allí que sea altamente probable que el docente omita la posibilidad de existencia de niños con altas capacidades intelectuales, desconociendo sus características y que probablemente por lo menos uno de esos niños o niñas atendidos podría estar dentro de este grupo; al mismo tiempo, se presume que no se ha considerado la capacitación del personal docente de educación inicial en esta área, por cuanto el personal directivo también pudiera desconocer la existencia de este 
tipo de situaciones educativas.

\subsection{Objetivo de Investigación}

A los fines del presente artículo se expondrá el primer objetivo específico:

- Identificar el conocimiento que poseen las docentes del Centro de Educación Inicial Los Retoñitos, ubicado en la Parroquia Alto Barinas del Municipio Barinas del Estado Barinas, sobre las características específicas de niños y niñas con Altas Capacidades Intelectuales en preescolares de 5 y 6 años de edad.

\subsection{Justificación de la Investigación}

Los docentes de educación inicial necesitan aprender a identificar oportunamente cuáles son las distintas capacidades y potencialidades de cada uno de los niños a su cargo, y así aplicar estrategias de trabajo diseñadas especialmente para proporcionar el pleno desarrollo de sus capacidades y potencialidades, además de sus talentos mediante la estimulación de sus intereses, creatividad; todo esto a través de un programa de aprendizaje auto dirigido.

Tomando en cuenta que los niños y niñas que presentan altas capacidades intelectuales no son un conjunto uniforme, Angulo (1996), plantea que:

...en un curso pueden cohabitar niños y niñas de inteligencia superior, media, baja, como seguramente pueden encontrarse con problemas de audición, visión, motricidad. En tales circunstancias la creatividad ni los criterios evaluativos pueden regirse por un patrón general y hay que tomar en cuenta además, que en ocasiones no solo importa el producto sino el esfuerzo ante las limitaciones para realizarlo. No todos pueden atenderse de igual manera y menos cuando a ciencia cierta se aprecian marcadas diferencias (pág. 21). 
De lo mencionado por el autor se plantea que el docente debe estar consciente que los niños son organismos evolutivos cada uno en distinto nivel, desarrollándose unos primero que otros, por lo que el maestro debe evitar promover el individuo conformista del mañana, evitar contener el pensamiento divergente y las inteligencias superiores, hasta llegar a anularlas por no ser entendidas, atendidas y fortalecidas como un hecho maravilloso que puede y debe construir al adulto del mañana que aporte y sea habitante activo de la sociedad que lo educa amorosa y responsablemente.

En tal sentido, es imprescindible que el docente tenga claro la existencia de este tipo de alumnos en su institución y así poder definir la intervención educativa que puedan requerir de forma temporal o permanente a lo largo de permanencia en el sistema educativo. La falta de preparación de los docentes puede originar, la calificación de un niño con gran potencial como flojo o niño problema, determinando su aislamiento y progresiva desaparición de las condiciones cognitivas superiores que tenía, robándoles al niño, niña y al docente también la posibilidad de ser compañeros de aventuras.

A partir de lo antes señalado se diseñó la presente investigación, la cual busca llamar la atención sobre un problema poco estudiado en Venezuela, fundamentalmente en lo referido a la capacitación del docente para detectar e intervenir educativamente al niño con capacidades cognitivas superiores, saber cómo tratarlo y comprometerlo emocionalmente con los desafíos que implican sumarse a la aventura de un encuentro más creativo en el aula.

\subsection{Referentes Teóricos}

\subsubsection{Altas Capacidades Intelectuales}

Se plantea entonces que los niños y niñas con altas capacidades intelectuales son aquellos que ostentan una capacidad y una forma de aprendizaje superior al de los niños de su edad, presentan mayor capacidad lógica, por lo cual razonan y aprenden más rápido. Adicionalmente, la 
capacidad verbal es más desenvuelta por lo que poseen un vocabulario más

amplio. El factor relevante es que presentan necesidades educativas, las cuales pueden variar dependiendo de sus factores internos y de los contextos en los cuales se aprende y se desarrollan. Estas necesidades dependen de su edad, los momentos del desarrollo humano del tipo afectivo y social.

En función de lo anterior se puede evidenciar que las altas capacidades intelectuales conceden al individuo un ritmo y una forma de aprendizaje distinto el cual generalmente no se ajustan al sistema de enseñanza regular, debido a que muchos de sus intereses y habilidades no se encuentran en los contenidos escolares, es decir, no se imparten en el sistema educativo; tales como el enfoque estratégico, creatividad, resolución de problemas, interrelación de conceptos de las diferentes asignaturas. En tal sentido, mucho de los intereses de esta población se dirigen a temas como paleontología, astronomía, física, química, biología, historia o filosofía no se ahondan en el currículo en los primeros niveles educativos.

Otro aspecto resaltante es, que entre la mayoría de los individuos con esta condición se presentan un desarrollo asincrónico, es decir, las funciones cognitivas se desarrollan de forma desigual, el desarrollo intelectual va por delante de su crecimiento. Adicionalmente, existe la disincronía social, por la sensación de ser diferentes al resto de sus compañeros, generando aislamiento en su propio mundo lo que puede confundirse con Síndrome de Asperger 0 encubrimiento de su potencial al intentar adaptarse. Adicionalmente, el desarrollo asincrónico puede hacer que algunos de los sujetos parezcan inmaduros y en ocasiones con problemas para escribir por presentar un desarrollo motriz no acorde con su desarrollo intelectual. Es necesario señalar que en algunas oportunidades factores ambientales tales como la pobreza, discriminación, barreras culturales, motivación, dificultades de aprendizaje entre otros evitan que se muestren y desarrolle los niveles de rendimiento a los que podría llegar en circunstancias óptimas. 
Por consiguiente, aunque este grupo de estudiantes no son uniformes en sus características se hace necesario que el docente maneje información sobre algunas de las cuales podrían presentar este tipo de individuos, así poder ser colaborador con los especialistas en el proceso de identificación, y tener disposición de afrontar la tarea que implica trabajar con ellos, y ser un ente que facilite potenciar un enriquecimiento del aprendizaje y el máximo desarrollo de sus capacidades individuales.

\section{Marco Metodológico}

El presente artículo responde al tipo descriptivo y de campo, la cual se refiere a la búsqueda de información directamente de la realidad, aplicando técnicas de recolección y análisis coherente con este propósito, es decir, el investigador se dirigirá al lugar para recopilar los datos necesarios. En relación con esto, Arias (2012), la define como: "la recolección de datos directamente de los sujetos investigados, o de la realidad donde ocurren los hechos (datos primarios), sin manipular o controlar variable alguna, es decir, el investigador obtiene la información, pero no altera las condiciones existentes" (pág. 31).

De tal forma que, el presente artículo responde a las necesidades del contacto directo con el personal docente del C.E.I. Los Retoñitos ubicado en la Parroquia Alto Barinas del Municipio Barinas del estado Barinas, los cuales se convierten en la población de estudio para la recolección de la información y serán descritos en detalle más adelante. Asimismo, el diseño de investigación fue de carácter descriptivo, por cuanto tiene como propósito fundamental describir las necesidades de capacitación de las docentes del C.E.I. Los Retoñitos, en cuanto a la atención educativa integral a niños que por sus características sociales y cognitivas pudiesen presentar Altas Capacidades Intelectuales.

Igualmente, para obtener la información requerida se tomó como población a las dieciocho (18) docentes de aula de los dos turnos (mañana y 
tarde) que laboraron durante el año escolar 2016-2017. En tal sentido, al ser una población finita y manejable, se tomó la muestra censal (totalidad de la población), es decir, la información fue obtenida a través de las dieciocho (18) docentes de aula que conforman la población del C.E.I. Los Retoñitos ubicado en la Parroquia Alto Barinas del Municipio Barinas del estado Barinas, durante el año escolar 2016-2017.

De esta manera, en relación con el objetivo planteado y la metodología seleccionada, se consideró necesario el uso de un cuestionario constituido por veinticinco (25) ítems de escalas alternativas impares, con el propósito de ver las tendencias de las respuestas emitidas siendo ellas: Siempre; Casi Siempre, A veces, Casi Nunca y Nunca; para recolectar la información a través de preguntas de respuestas fijas conocido como Escala tipo Likert.

Del mismo modo, la validación en el presente instrumento se obtuvo a través de la valoración del juicio de expertos, constituidos por tres (3) docentes universitarios de gran experiencia en investigación quienes se desempeñan como personal académico ordinario de la Universidad Pedagógica Experimental Libertador en su Instituto de Mejoramiento Profesional del Magisterio de la Extensión Académica Barinas. Igualmente, la confiabilidad se obtuvo a partir de una muestra piloto realizada a diez (10) sujetos con las mismas características laborales y académicas de los individuos objeto de estudio, calculado por el coeficiente de determinación de consistencia interna Alfa de Cronbach, generando un resultado de 0.89 de confiabilidad; lo cual demuestra que esta es Muy Alta. Posteriormente se analizaron los datos obtenidos a través de la estadística descriptiva la cual se encarga de caracterizar un conjunto de datos.

\section{Análisis e Interpretación de Resultados}

El presente apartado consiste en cuantificar, analizar, tabular e interpretar el conjunto de resultados obtenidos en la aplicación del instrumento 
conformado por veinticinco (25) ítems o preguntas cerradas; aplicado a las dieciocho (18) docentes del nivel educación inicial del C.E.I. Los Retoñitos con el fin determinar las necesidades de capacitación que presentan sobre las Altas Capacidades Intelectuales, determinándose la frecuencia de ocurrencia de cada respuesta emitida por estas y su porcentaje correspondiente, reflejando los datos en cuadros y gráficos representados por diagramas de tortas, acompañados de un análisis de los datos.

En esta sección se dan a conocer los resultados correspondientes a los ítems números 5, 11, 21 y 25 del cuestionario diseñado para identificar los elementos más relevantes que requieren ser conocidos en relación con la variable denominada Necesidades de Capacitación Docente.

En cuanto al ítem 5 dirigido al conocimiento sobre el manejo de información especializada que tienen las docentes sobre las características del estudiante con Altas Capacidades Intelectuales, como se puede evidenciar en el Cuadro N.. 1 y Gráfico N.1, el 5,56\% de los encuestados manifestó que "a veces" maneja dicha información, mientras que un 38,89\% declaró que "casi nunca" lo hace, y el $55,56 \%$ de los encuestados reflejan que "nunca" manejan esta información.

Esto es un dato que llama la atención por cuanto el manejo de información especializada sobre las características del estudiante con Altas Capacidades Intelectuales representa un elemento fundamental para su identificación o, por lo menos, sospechar de su existencia para poder levantar un informe y remitir a la evaluación del especialista correspondiente.

He allí la importancia de que el docente de aula en el nivel de Educación Inicial maneje información especializada para responder adecuadamente a las situaciones educativas individuales que pudieran presentar algunos estudiantes con Altas Capacidades Intelectuales, permitiendo dar mayor posibilidad para una identificación temprana con niños que presenten esta condición. Claro está, la importancia de esta situación radica en lo que, como 
señala Apraiz (1996): "se considera que es necesario realizar una identificación de los sujetos, no con la finalidad de etiquetarlos, sino para estar en condiciones idóneas de poder determinar las necesidades educativas que presentan y dar respuesta a las mismas" (pág. 76).

Cuadro N. 1. Distribución de Frecuencia de la Variable Necesidades de Capacitación Docente, Dimensión Carencias e Indicador Conocimiento con relación al Ítem 5: Tengo conocimientos especializados sobre quiénes son los estudiantes que presenta Altas Capacidades Intelectuales.

\begin{tabular}{lcc}
\hline Alternativa & Frecuencia & $\%$ \\
\hline SIEMPRE & 0 & 0,00 \\
CASI SIEMPRE & 0 & 0,00 \\
A VECES & 1 & 5,56 \\
CASI NUNCA & 7 & 38,89 \\
NUNCA & 10 & 55,56 \\
\hline TOTAL & $\mathbf{1 8}$ & $\mathbf{1 0 0}$ \\
\hline
\end{tabular}

Fuente: La Autora (2017).

Gráfico N. 1. Distribución porcentual de las respuestas aportadas en el ítem 5 del cuestionario aplicado a las Docentes de Educación Inicial del C.E.I. Los Retoñitos. Variable: Necesidades de Capacitación Docente.

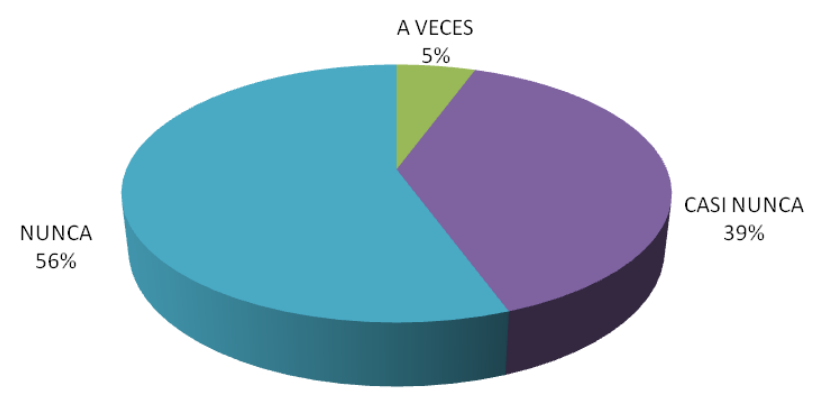

Fuente: La Autora (2017).

El ítem 11 el cual señala sobre el conocimiento que poseen las docentes 
sobre el método para diagnosticar a los estudiantes con altas capacidades intelectuales; se puede evidenciar en el Cuadro N. -2 y el Gráfico N.․ 2 que el $38,89 \%$ de los encuestados reflejan que "a veces" manejan esta información, y el $61,11 \%$ reflejan que "casi nunca" la manejan, es decir, que la mayoría de ellas casi nunca conocen el método para diagnosticar a los estudiantes con Altas Capacidades Intelectuales, siendo un factor de gran importancia debido a que es el docente quien pasa una gran cantidad de tiempo con los estudiantes teniendo la oportunidad de observar cómo se desenvuelve el alumno en el contexto escolar.

En función de lo señalado anteriormente, se hace necesario que el docente de Educación Inicial obtenga la capacitación que les permita diagnosticar tempranamente a los estudiantes con Altas Capacidades Intelectuales para poder brindarle la atención especializada en función del desarrollo máximo de su potencial individual.

Cuadro N. 2. Distribución de Frecuencia de la Variable Necesidades de Capacitación Docente, Dimensión Carencias e Indicador Conocimiento con relación al Ítem 11: Conozco cual es el método para diagnosticar a los estudiantes con altas capacidades intelectuales.

\begin{tabular}{lcc}
\hline Alternativa & Frecuencia & $\%$ \\
\hline SIEMPRE & 0 & 0,00 \\
CASI SIEMPRE & 0 & 0,00 \\
A VECES & 7 & 38,89 \\
CASI NUNCA & 11 & 61,11 \\
NUNCA & 0 & 0,00 \\
\hline TOTAL & $\mathbf{1 8}$ & $\mathbf{1 0 0}$
\end{tabular}

Fuente: La Autora (2017). 
Gráfico N. 2. Distribución porcentual de las respuestas aportadas en el ítem 11 de la aplicación del cuestionario a las Docentes de Educación Inicial del C.E.I. Los Retoñitos. Año 2017. Variable: Necesidades de Capacitación Docente.

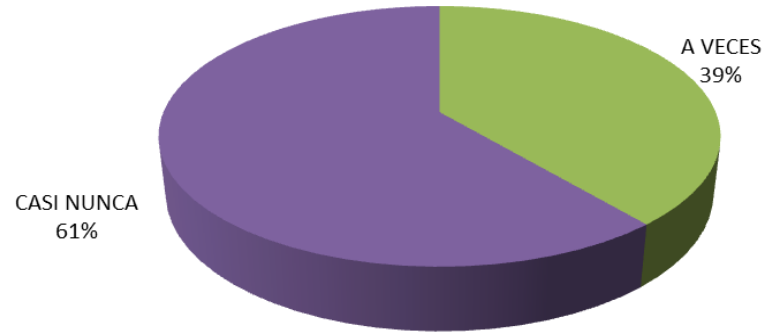

Fuente: La Autora (2017).

El ítem 21, tiene como objeto recoger información sobre si el docente de educación inicial identifica las estrategias didácticas para la atención educativa del estudiante con Altas Capacidades Intelectuales, en este sentido, se observa en el Cuadro N. 3 y en el Gráfico N. .3 , como el 83,33\% de las encuestadas manifiestan que "casi nunca" identifican cuales son las estrategias didácticas para la atención educativa a este tipo de alumnado, así como el $16,67 \%$ señala que "a veces" las identifica, haciéndose fundamental orientar y capacitar a las docentes de educación inicial en lo referente a la organización de estrategias didácticas que permitan dar respuestas educativas que colaboren en la superación de las barreras para el aprendizaje con la participación de todo el alumnado para el beneficio de la totalidad del grupo, no únicamente al alumnado con $(\mathrm{ACl})$.

De allí la importancia de ofrecer al docente las herramientas que le permitan identificar las estrategias didácticas para la atención educativa del estudiante con Altas Capacidades Intelectuales y así lograr la intervención que permita dar respuesta a necesidades educativas y facilitando el máximo desarrollo de sus capacidades. 
Cuadro N. 3. Distribución de Frecuencia de la Variable Necesidades de Capacitación Docente, Dimensión Carencias e Indicador Habilidades con relación al Ítem 21: Identifico estrategias didácticas para la atención educativa del estudiante con altas capacidades intelectuales.

\begin{tabular}{lcc}
\hline Alternativa & Frecuencia & $\%$ \\
\hline SIEMPRE & 0 & 0.00 \\
CASI SIEMPRE & 0 & 0.00 \\
A VECES & 3 & 16.67 \\
CASI NUNCA & 15 & 83.33 \\
NUNCA & 0 & 0.00 \\
\hline TOTAL & $\mathbf{1 8}$ & $\mathbf{1 0 0}$ \\
\hline
\end{tabular}

Fuente: La Autora (2017).

Gráfico N. 3. Distribución porcentual de las respuestas aportadas en el ítem 21 de la aplicación del cuestionario a las Docentes de Educación Inicial del C.E.I. Los Retoñitos. Año 2017. Variable Necesidades de Capacitación Docente.

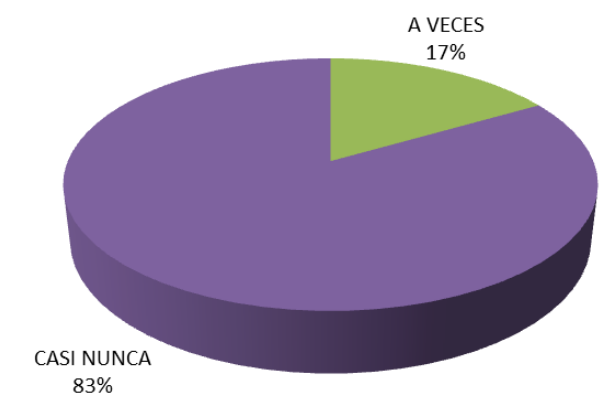

Fuente: La Autora (2017).

\section{Conclusiones y Recomendaciones}

\subsection{Conclusiones}

Atendiendo a los resultados del estudio se concluye lo siguiente:

En relación con el objetivo que busca identificar el conocimiento que poseen las docentes del C.E.I. Los Retoñitos sobre las Altas Capacidades 
Intelectuales en niños y niñas de 5 y 6 años de edad, se evidencia que estas no se encuentran preparados para atender ni responder adecuadamente a los requerimientos de esta población, además desconocen cuáles son las características generales más comunes de esta población, al igual que las medidas para atenderlos. Esto se evidencia en los porcentajes promedio de respuesta de los encuestados, por cuanto entre el $36 \%$ de las encuestadas manifiestan que "casi nunca" identifican las características específicas de los niños y niñas con Altas Capacidades Intelectuales de 5 y 6 años de edad, lo cual evidencia una necesidad de capacitar en esta área.

En tal sentido, se consideran las docentes como un factor relevante por lo que son los entes sociales que podrían reconocer las características que forman parte de la gama de rasgos que una persona puede presentar para considerarlo como sujeto con ( $\mathrm{ACl}$ ); paralelamente, ellas las que tienen la responsabilidad y deberían tener la capacidad dentro de las instituciones educativas y las aulas de clase de reconocer y hacer sobresalir a aquellos estudiantes que no han sido reconocidos por su alto potencial.

Es pertinente entonces mencionar la importancia del conocimiento que deben poseer los docentes en los procesos de identificación en edades tempranas; tal como señala Benito (2008b):

La importancia de la identificación temprana como en cualquier otro nivel de la educación especial es primordial. En el caso de los niños superdotados se ha constatado que las explicaciones sobre las diferencias entre la promesa y la realización del superdotado apuntan a las diferencias sustanciales en los entornos tempranos favorables y sus factores familiares y oportunidades educativas y profesionales (pág. 24).

\subsection{Recomendaciones}

En función al objetivo formulado en la investigación se realizan las siguientes recomendaciones:

Se recomienda organizar talleres para la preparación dirigido al docente 
de educación inicial que ayude a fortalecer el conocimiento que poseen sobre las características generales y específicas de los niños y niñas con Altas Capacidades Intelectuales. Asimismo, se recomienda establecer un equipo multidisciplinario de docentes para el uso de las técnicas de detecciónintervención para identificar las altas capacidades intelectuales en los niños y niñas de 5 y 6 años de edad.

Igualmente, se debe incentivar al personal docente en el uso del enriquecimiento curricular como estrategia didáctica para la atención educativa, de allí que se insta al personal directivo realizar seguimiento en el aula a los docentes para apoyarlo en su práctica pedagógica y facilitar el uso de adaptaciones curriculares como estrategias didácticas para la atención educativa en la atención de los niños y niñas de 5 y 6 años de edad con altas capacidades intelectuales.

\section{Referencias}

Apraiz, J. (coord.) (1996). Educación del alumnado con altas capacidades.

Victoria, España: Servicio central de publicaciones del Gobierno Vasco. Artiles, C. (2006). La atención educativa al alumnado con Altas Capacidades Intelectuales desde un programa institucional a largo plazo financiado y dirigido por la administración educativa de la Comunidad Autónoma de Canarias. Barcelona, España: Universidad de las Palmas de Gran Canaria. Recuperado de: https://www.eoepmolina.es/wp-content/gallery/AACC/A01Artiles.pdf

Arias, F. (2012). EI Proyecto de Investigación. Sexta Edición. Caracas, Venezuela: Editorial Episteme, C.A.

Angulo, C. (1996). La Psicología Cotidiana. Caracas, Venezuela: Fondo de Publicaciones de Instituto Nacional del Menor.

Barrios, H. (2009a.b). Identificación y educación de niños y niñas superdotados; Propuesta de investigación para la identificación 
subjetiva y la educación integral de calidad de los niños y las niñas superdotadas. Venezuela: Fundación de Investigación y Educación de la Salud Holística, FIELSALUH.

Benavides, M., Maz, A., Castro, E. \& Blanco, R. (2004a,b). La Educación de Niños con Talento en Iberoamérica. Oficina Regional de Educación para América Latina y el Caribe, ISBN: 956-8302-34-4. Santiago, Chile:

Editorial Trineo S.A. Recuperado de:

http://unesdoc.unesco.org/images/0013/001391/139179s.pdf

Blanco, R., Ríos, C., \& Benavides, M. (2004). La educación de niños con talento en Iberoamérica. Oficina Regional de Educación para América Latina y el Caribe. Santiago, ISBN: 956-8302-34-4. Chile: Editorial Trineo S.A. Recuperado de:

http://unesdoc.unesco.org/images/0013/001391/139179s.pdf

Benito, Y. (2008a.b). Identificación temprana: identificación del niño superdotado en el hogar. VII Congreso BIENAL de la FICOMUNDYT, ISSN Internet: 1695-7075, ISSN formato papel: 1134-1548. España: Edita Ideación n.ำ 28, págs. 285-320. Recuperado de:

http://www.templetonfellows.org/projects/docs/ficomundyt.pdf

Freeman, J. (dir) (1988). Los niños superdotados. Madrid, España: Santillana.

Matos, Y., Vethencourt, M., Peña, M., \& Briceño, M. (2018). Inteligencias que Desarrolla el Estudiante Jugador de Ajedrez. Revista Scientific, 3(8), 248-271. Recuperado de:

https://doi.org/10.29394/Scientific.issn.2542-2987.2018.3.8.13.248-271 Ministerio de Educación (1997). Conceptualización y Política para el Desarrollo del Talento. Caracas, Venezuela: Ediciones UPEL-IMPM, págs. 195-256. 


\section{Adriana Josefina Ovalles Villegas \\ e-mail: aovalles40@gmail.com}

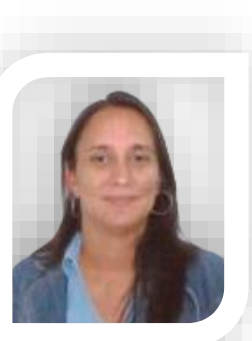

Nacida en Caracas, Venezuela. Licenciada en Educación Especial primera de su promoción en la Universidad Nacional Experimental de los Llanos Occidentales Ezequiel Zamora, Maestrante en Orientación Educativa de la Universidad Pedagógica Experimental Libertador en su Instituto de Mejoramiento Profesional del Magisterio. Docente Instructor a Tiempo Completo de la Universidad Pedagógica Experimental Libertador en su Instituto de Mejoramiento Profesional del Magisterio, adscrita a la Extensión Académica Barinas. Entre sus temáticas de investigación destacan: Las Necesidades Educativas Especiales de Niños con Altas Capacidades. Actualmente se desempeña como Responsable de Evaluación y Responsable de Bienestar Estudiantil. Publica artículos científicos en revistas científicas y participa como Conferencista, Ponente y Tallerista en actividades académicas.

El contenido de este manuscrito se difunde bajo una Licencia de Creative Commons ReconocimientoNoComercial-Compartirlgual 4.0 Internacional 